\title{
Impact Analysis of Accident Using AI
}

\section{Divya Mishra ${ }^{1}$, Dr Shashank Singh ${ }^{2}$, Dr. Faiyaz Ahamad ${ }^{3}$}

${ }^{1}$ M.Tech. Scholar, Department of Computer Science and Engineering, Integral University, Lucknow , India ${ }^{2}$ Assistant Professor, Department of Computer Science and Engineering, Integral University, Lucknow , India

\section{Article Info}

Volume 7, Issue 4

Page Number: 397-405

\section{Publication Issue :}

July-August-2021

\section{Article History}

Accepted : 20 July 2021

Published : 27 July 2021

\section{ABSTRACT}

There are numerous inventories in vehicle enterprises to plan and fabricate security measures for autos, yet car crashes are unavoidable. There is countless mishaps winning in all metropolitan and country regions. Examples associated with various conditions can be identified by fostering a precise forecast models which will be equipped for programmed partition of different inadvertent situations. These group will be valuable to forestall mishaps and foster security measures. We accept to procure greatest conceivable outcomes of mishap decrease utilizing low spending assets by utilizing some logical measures. There is a gigantic effect on the general public because of auto collisions where there is an extraordinary expenses of fatalities and wounds. Lately, there is an increment in the investigate thoughtfulness regarding decide the fundamentally influence the seriousness of the drivers wounds which is caused because of the street mishaps. Exact and complete mishap records are the premise of mishap investigation .the compelling utilization of mishap records relies upon certain variables, similar to the exactness of the information, record maintenance, and information examination. There is numerous methodologies applied to this situation to examine this issue. In this examination paper Open CV apparatus is utilized to mishap sway investigation. A new report delineated that the private and shopping locales are more perilous than town areas.as may have been anticipated, the frequencies of the setbacks were higher close to the zones of home perhaps in view of the greater openness .An examination uncovered that the loss rates among the neighborhoods are delegated generally denied and altogether higher than those from moderately rich regions.

Keywords : fabricate security, street mishaps, Open CV, methodologies.

\section{INTRODUCTION}

Plan and control of traffic by cutting edge frameworks come in see as the significant need. Suspicion on the dangers in rush hour gridlock and the guidelines and intercessions toward the finish of these suppositions will decrease the street mishaps. A presumption framework which will be set up with accessible information and new dangers will be invaluable. Information mining idea had been thought of by expanding and capacity of information in the advanced stage. Information mining includes the investigations which will find data from precise and

Copyright: () the author(s), publisher and licensee Technoscience Academy. This is an open-access article distributed under the terms of the Creative Commons Attribution Non-Commercial License, which permits unrestricted non-commercial use, distribution, and reproduction in any medium, provided the original work is properly cited 
intentional information structures got from cluttered and good for nothing information. AI which is sub-part of manmade brainpower supplies learning of PC exploiting information distribution centers .

Suspicion capacities of PC frameworks have progressed in case of AI. Usage of AI is a broad and practical technique for taking credible choices by utilizing experience. AI can accomplish extricate data from information and utilize measurable technique. The expenses of fatalities and wounds because of car crashes extraordinarily affect the general public. As of late, specialists have given expanding consideration to deciding components that essentially influence seriousness of driver wounds brought about by car crashes [29][30]. There are a few methodologies that specialists have utilized to examine this issue.

These incorporate neural organization, settling rationale plan, log-direct model, fluffy ART maps, etc. Applying information mining strategies to display car crash information records can assist with understanding the attributes of drivers' conduct, street condition and climate condition that were causally associated with various injury seriousness. This can help chiefs to detail better traffic wellbeing control approaches. Roh et al. [22] outlined how factual strategies dependent on coordinated charts, built over information for the new period, might be valuable in displaying traffic fatalities by contrasting models indicated utilizing coordinated diagrams with a model, in view of out-of-test estimates, initially created by Peltzman [23]. The coordinated charts model beat Peltzman's model in root mean squared estimate mistake.

The always expanding gigantic measure of information, gathered and put away in enormous and various information bases, has far surpassed human capacity for cognizance without the utilization of integral assets [3]. Thus, significant choices are regularly made put together not with respect to the data rich information put away in data sets but instead on a chief's instincts because of the absence of apparatuses to remove the important information installed in the huge measures of information [3]. This is the reason information mining has gotten extraordinary consideration lately.
Information mining includes an incorporation of procedures from numerous controls like data set innovation, insights, AI, elite figuring, design acknowledgment, neural organizations, information perception, data recovery, picture and sign handling, and spatial information investigation [3][19]. General information mining standards, including Associations, Sequential Patterns, Classifications, Predictions, and Clustering, can be applied to numerous spaces. Arrangement calculations give intriguing outcomes from an enormous arrangement of information ascribes.

The expenses of fatalities and wounds because of car crashes extraordinarily affect society. The World Health Organization [14] predicts that street impacts will bounce from the 10th driving reason for death in 2004 to the fifth in 2030. Many exploration works are focusing on breaking down different accident related elements which increment the passing proportion. Comparable to this, deadly severities came about because of street car crash are one of the spaces of concern. Out of all street related components the way of impact impacts the lethal rate. As the size of these mishap data sets increments quickly both spatially and transiently, it is a serious test to dissect and extricate valuable data from them without utilizing progressed information examination instruments.

In this paper section I contains the introduction, section II contains the objective details, section III contains the details about problem statement, section IV contains the literature review details, section $\mathrm{V}$ shows methodology details, VI shows the architecture, section VII provide result of this paper and section VIII provide the conclusion of this research paper.

\section{Objectives}

In this research paper the main objective of the impact analysis of accident using AI system is:

- Analyze the previously occurred accidents in the locality which will help us to determine the most accident-prone area and help us to set up the immediate required help for them.

- Solving problem of road accident using Artificial Intelligence and Image Processing. 
- Verify the relationships among accident type, and injury severity.

- Analyse the impact of the identified key factors on accident and fatality occurrence.

- Determine significant risk factors associated with each accident type that are highly correlated to injury severity.

- It is helpful for insurance company

- It is helpful for police investigation

\section{Problem Statement}

There are several problems with current practices for prevention of the accidents occurred in the localities. The road accident's to lead to loss of human life and/or incapacitation. It was noted in road accidents, that most cases of results are death of people and people lose their loved ones. Some people prefer to proceed with a reimbursement claim. In these sort of cases catching the location of the occasions with a photo can be useful. You can snap a couple of photos of the mishap which include your vehicle harms, substantial wounds and so forth, and fill in as legitimate verification during guarantee settlement.

\section{LITERATURE REVIEW}

Ossenbruggen et al. [24] utilized a strategic relapse model to recognize genuinely huge elements that foresee the probabilities of accidents and injury crashes targeting utilizing these models to play out a danger evaluation of a given district. These models were elements of variables that depict a site by its territory use action, side of the road configuration, utilization of traffic light gadgets and traffic openness. Their examination showed that town destinations are less dangerous than private and shopping locales.

Abdalla et al. [25] contemplated the connection between loss frequencies and the distance of the mishaps from the zones of home. As might have been expected, the setback frequencies were higher closer to the zones of home, conceivably because of higher openness. The investigation uncovered that the loss rates among occupants from regions named moderately denied were fundamentally higher than those from generally princely regions.
Miaou et al. [26] contemplated the factual properties of four relapse models: two traditional straight relapse models and two Poisson relapse models as far as their capacity to demonstrate vehicle mishaps and parkway mathematical plan connections. Street and truck mishap information from the Highway Safety Information System (HSIS) have been utilized to delineate the utilization and the constraints of these models. It was shown that the customary direct relapse models come up short on the distributional property to portray satisfactorily irregular, discrete, nonnegative, and regularly inconsistent vehicle mishap occasions out and about. The Poisson relapse models, then again, have the vast majority of the attractive measurable properties in fostering the connections.

Abdelwahab et al. considered the 1997 mishap information for the Central Florida region [2]. The investigation zeroed in on vehicle mishaps that happened at signalized convergences. The injury seriousness was partitioned into three They looked at the presentation of Multi-layered Perceptron (MLP) and Fuzzy ARTMAP, and tracked down that the MLP order precision is higher than the Fuzzy ARTMAP. Levenberg-Marquardt calculation was utilized for the MLP preparing and accomplished 65.6 and 60.4 percent arrangement exactness for the preparation and testing stages, separately. The Fuzzy ARTMAP accomplished an order precision of 56.1 percent. Yang et al. utilized neural organization way to deal with recognize more secure driving examples that have less odds of causing demise and injury when a fender bender happens [17]. They played out the Cramer's V Coefficient test [18] to recognize huge factors that cause injury to lessen the components of the information. Then, at that point, they applied information change technique with a recurrence based plan to change all out codes into mathematical qualities. They utilized the Critical Analysis Reporting Environment (CARE) framework, which was created at the University of Alabama, utilizing a Backpropagation (BP) neural organization.

They utilized the 1997 Alabama highway liquor related information, and further contemplated the loads on the prepared organization to get a bunch of controllable reason factors that are likely causing the injury during an accident. The objective variable in their examination had two classes: injury and non-injury, in which injury class included 
fatalities. They found that by controlling a solitary variable (like the driving velocity, or the light conditions) they possibly could diminish fatalities and wounds by up to $40 \%$

Sohn et al. applied information combination, outfit and bunching to improve the precision of individual classifiers for two classifications of seriousness (substantial injury and property harm) of street auto collisions [15]. The individual classifiers utilized were neural organization and choice tree. They applied a grouping calculation to the dataset to isolate it into subsets, and afterward utilized every subset of information to prepare the classifiers. They found that arrangement dependent on grouping works better if the variety in perceptions is generally enormous as in Korean street auto collision information. Mussone et al. utilized neural organizations to examine vehicle mishap that happened at crossing points in Milan, Italy [12]. They picked feed-forward MLP utilizing BP learning.

The model had 10 info hubs for eight factors (day or night, traffic streams circling in the convergence, number of virtual struggle focuses, number of genuine clash focuses, kind of crossing point, mishap type, street surface condition, and climate conditions). The yield hub was called a mishap file and was determined as the proportion between the quantity of mishaps for a given crossing point and the quantity of mishaps at the most hazardous convergence. Results showed that the most noteworthy mishap record for running over of passerby happens at non-signalized crossing points at evening.

Dia et al. utilized certifiable information for fostering a multifaceted MLP neural organization road episode discovery model [5]. They looked at the presentation of the neural organization model and the episode identification model in procedure on Melbourne's interstates. Results showed that neural organization model could give quicker and more solid episode discovery over the model that was in activity. They additionally found that inability to give speed information at a station could essentially weaken model execution inside that part of the road.

Shankar et al. applied a settled rationale detailing for assessing mishap seriousness probability adapted on the event of a mishap [14]. They found that there is a more prominent likelihood of clear injury or crippling injury/casualty comparative with no apparent injury if at any rate one driver didn't exercise a self control framework at the hour of the mishap. Kim et al. fostered a log-direct model to explain the job of driver attributes and practices in the causal arrangement prompting more extreme wounds. They found that liquor or medication use and absence of safety belt use significantly increment the chances of more serious accidents and wounds [8].

Abdel-Aty et al. utilized the Fatality Analysis Reporting System (FARS) crash data sets covering the time of 19752000 to dissect the impact of the expanding number of Light Truck Vehicle (LTV) enlistments on lethal point impact patterns in the US [1]. They explored the quantity of yearly fatalities that came about because of point crashes just as impact setup (vehicle, vehicle LTV, LTV-vehicle, and LTVLTV). Time arrangement demonstrating results showed that fatalities in point crashes will increment in the following 10 years, and that they are influenced by the normal in general increment of the level of LTVs in rush hour gridlock.

Bedard et al. applied a multivariate calculated relapse to decide the free commitment of driver, crash, and vehicle qualities to drivers' casualty hazard [3]. They tracked down that expanding safety belt use, diminishing pace, and lessening the number and seriousness of driver-side effects may forestall fatalities. Evanco led a multivariate populace based measurable examination to decide the connection among fatalities and mishap warning occasions [6]. The investigation exhibited that mishap warning time is a significant determinant of the quantity of fatalities for mishaps on provincial streets.

Ossiander et al. utilized Poisson relapse to investigate the relationship between the deadly accident rate (lethal accidents per vehicle mile voyaged) and as far as possible increment [13]. They tracked down that as far as possible increment was related with a higher deadly accident rate and more passings on expressways in Washington State.

Handan et.al [4] contrasted strategic relapse model and arrangement tree technique in deciding social-segment hazard factors which have influenced discouragement status of ladies in discrete post pregnancy periods. They suggested that Classification tree strategy gives more data 
with detail on analysis by assessing a ton of hazard factors together than calculated relapse model.

Chang et.al [2] applied non-parametric order tree methods to examine Taiwan mishap information from the year 2001. They fostered a CART model to discover the connection between injury seriousness and driver/vehicle attributes, roadway/climate factors, and mishap factors.

Yong Soo Kim [11] looked at the presentation of information mining and factual strategies by changing the quantity of autonomous factors, the sorts of free factors, the quantity of classes of the free factors, and the example size. The outcomes have shown that the fake neural organization execution improved quicker than that of different techniques as the quantity of classes of absolute variable expanded.

I-Cheng et.al [5] explored the precision of information mining strategies viz. discriminant examination, calculated relapse, Bayes classifier, closest neighbor, counterfeit neural organizations, and grouping trees in breaking down clients' default credit installments in Taiwan and looks at the prescient exactness of likelihood of default among six information mining techniques. Their outcomes uncover that fake neural organization is the one in particular that can precisely appraise the genuine likelihood of default credit installments.

Weimin et.al [10] showed that the half breed SVM method having better ability of catching nonlinear relationship among factors and had best characterization rate than CART, MARS and SVM while examining the Visa information.

Nojun et.al [9] broke down the limit of Mutual Information Feature Selector (MIFS) and proposed a strategy to beat this restriction. Isabelle et.al [6] talked about the nuts and bolts of highlight determination and summed up the means to tackle an element choice issue. The execution of different element choice calculations have been examined in [15]. Next area sums up the insights regarding the preparation informational collection.

\section{Methodology}

Most past techniques have planned explicit finders utilizing various highlights for every one of these three classes. The methodology we guarantee here varies from these current methodologies in that we propose a solitary learning based discovery structure to identify every one of the three significant classes of items. To additionally improve the speculation execution, we propose an article sub arrangement strategy as a methods for catching the intraclass variety of items.

\section{a. Generic Object Detection}

Object location is a difficult however significant application in the PC vision local area. It has accomplished effective results in numerous viable applications, for example, face recognition and walker discovery. Complete overview of item recognition can be found in. This segment momentarily surveys a few conventional item identification strategies. These systems accomplish great location results on inflexible item classes. Be that as it may, for object classes with a huge intra-class variety, their recognition execution tumbles down drastically. As of late, another location structure which uses totaled channel highlights (TCH) and an AdaBoost classifier has been proposed in. This structure utilizes thorough slidingwindow search to recognize objects at multi-scales. It has been adjusted effectively for some reasonable applications.

\section{b. Proposed Solution}

We propose a solitary learning based discovery system (SLDS) to distinguish every one of the three significant classes of items. The proposed system comprises of a thick component extractor and finders of these three classes. When the thick highlights have been removed, these highlights are imparted to all indicators. The upside of utilizing one regular structure is that the identification speed is a lot quicker, since all thick highlights need just to be assessed once in the testing stage. The proposed structure presents spatially pooled highlights as a piece of amassed channel highlights to improve the component power to commotions and picture disfigurements. To additionally improve the speculation execution, we propose an item sub arrangement strategy as a methods for catching the intra-class variety of articles. 
Models are made utilizing mishap information records which can assist with understanding the attributes of numerous highlights. In this we are attempt to make a module in which we study the Impact brought about by street mishap whether it is minor or major by utilizing Image processing. The model is performed to distinguish measurably huge components which can have the option to anticipate the probabilities of accidents and injury that can be utilized to play out a danger factor and diminish it. Here the street mishap study is finished by investigating some information by giving a few questions which is applicable to the examination. The inquiries like what is the most hazardous opportunity to drive, what parts of mishaps happen in country, metropolitan and different regions. What is the pattern in the quantity of mishaps that happen every year, do mishaps in high velocity limit regions have more losses, etc.

\section{Convolutional Neural Networks (CNN)}

In this research paper Convolutional Neural Networks $(\mathrm{CNN})$ is one of the variations of neural organizations utilized vigorously in the field of Computer Vision. It gets its name from the sort of covered up layers it comprises of. The secret layers of a CNN regularly comprise of convolutional layers, pooling layers, completely associated layers, and standardization layers. Here it just implies that as opposed to utilizing the typical initiation capacities characterized above, convolution and pooling capacities are utilized as actuation capacities. To comprehend it exhaustively one requirements to comprehend what convolution and pooling are. Both of these ideas are acquired from the field of Computer Vision. Step utilized in CNN calculation is:

Step 1: Convolution Operation. ...

- $\quad$ Step 1(b): ReLU Layer. ...

- $\quad$ Step 2: Pooling....

- Step 3: Flattening. ...

- Step 4: Full Connection. ...

- Step 1 - Convolution Operation. ...

- Step 1(b): The Rectified Linear Unit (ReLU) ...

- Step 2 - Max Pooling.
VI. ARCHITECTURE

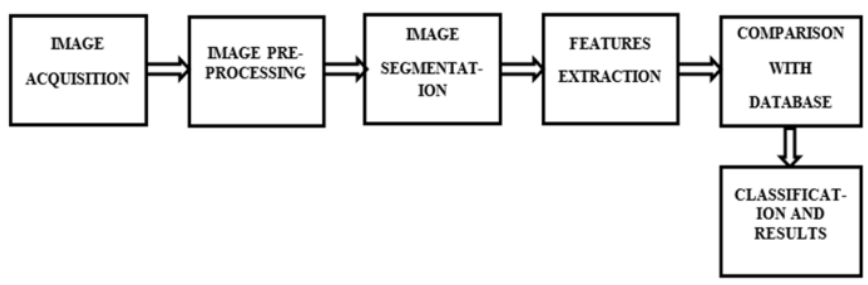

Figure 1: Architecture diagram

VII. Result

Object detection in computer vision. Object detection is the process of finding instances of real-world objects such as impact analysis of accident using artificial intelligence. Accident impact detection algorithms typically use extracted features and learning algorithms to recognize accident impact analysis like low accident impact or major accident impact. There is a need to play out a far reaching investigation that expects to comprehend the connection between the impact factors and car accident results. The commitment of this investigation lies in the advancement of model to break down auto collision information and to foresee the injury seriousness of car crashes Analyze the sort of mishap. In this examination, we introduced an imaginative methodology for fender bender forecast. Methodologically, we showed a learning strategy installed inside a multivariate model can be utilized to distinguish connection between the inspected factors and the car accidents. Future utilizations of this methodology can possibly give bits of knowledge into fundamental inquiries in regards to street spatial and worldly powerful capacity and functional inquiries with respect to countermeasures. Mishap sway identification is a PC innovation identified with PC vision and picture handling that arrangements with recognizing occasions of semantic objects of a specific class (like people, structures, vehicles) in computerized pictures.

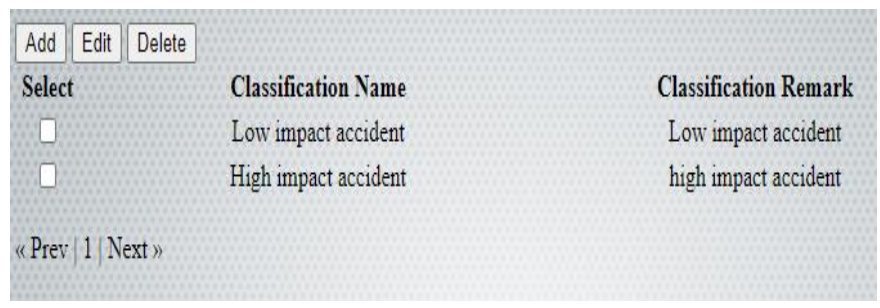

Figure 2: Accident Category Classification 


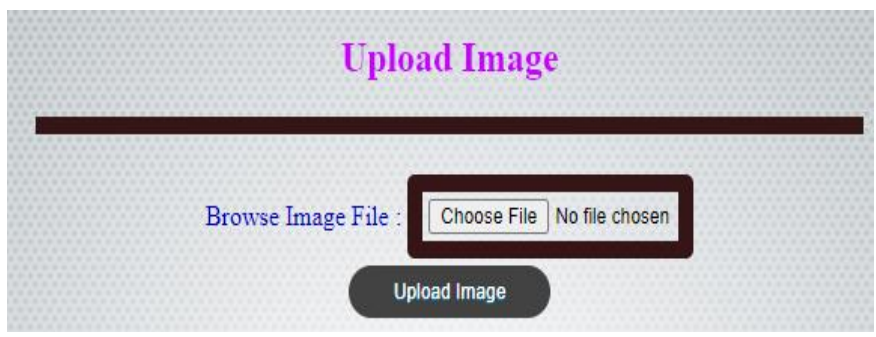

Figure 3: Training data set by uploading accident images

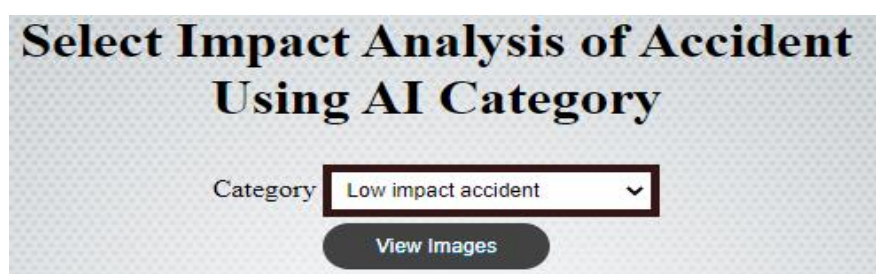

Figure 4: Admin can view trained data set

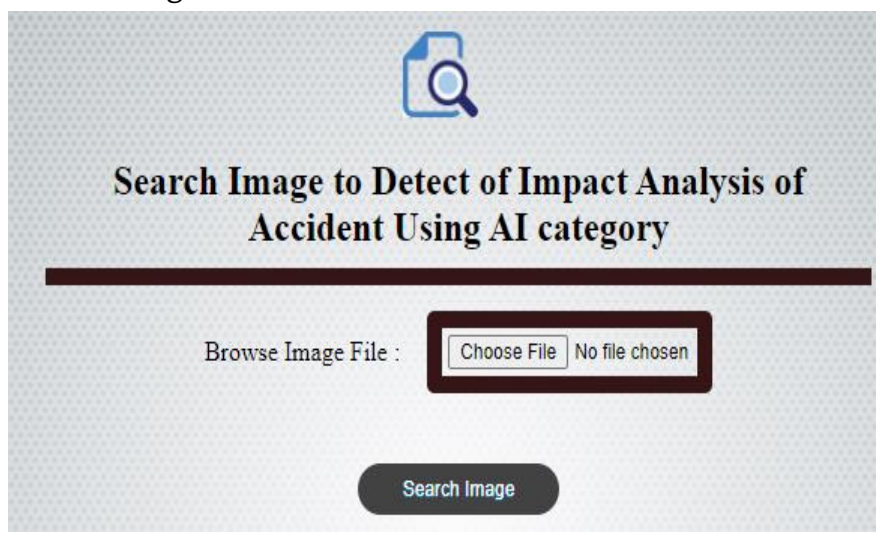

Figure 5: User can check accident impact analysis by uploading accident images

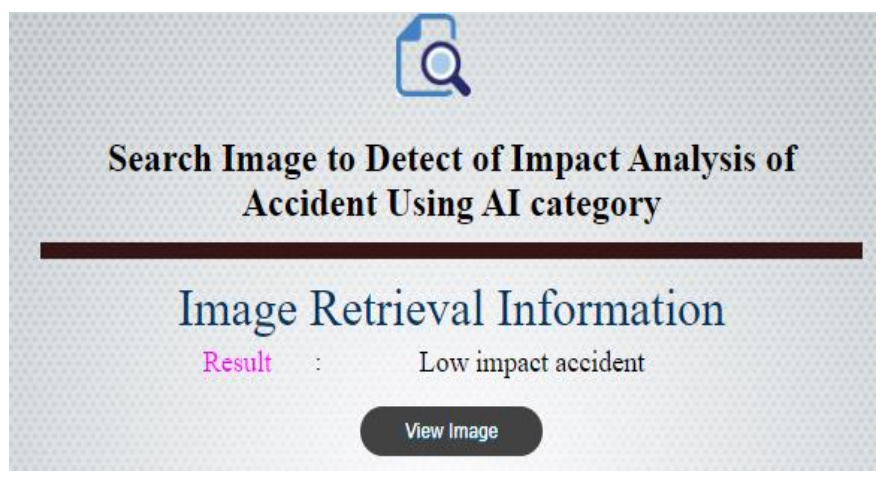

Figure 6: Showing result low impact analysis

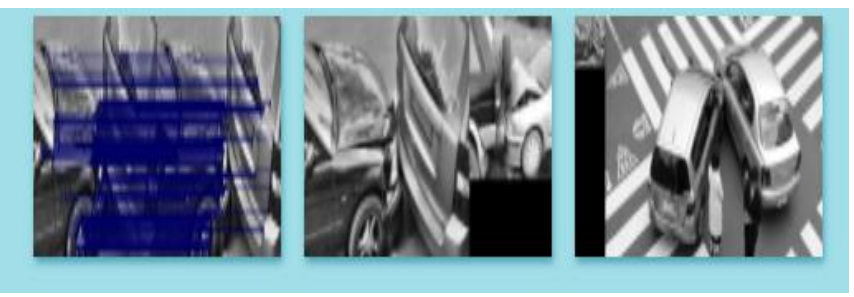

Figure 7: Showing image comparison between images

\section{CONCLUSION \& FUTURE SCOPE}

This paper incorporates car accidents are a major worry of general society, offices, and strategy creators and result in innumerable fatalities and wounds, there is a need to play out a complete examination that plans to comprehend the connection between the impact factors and car accident results. The commitment of this investigation lies in the advancement of model to break down car crash information and to anticipate the injury seriousness of auto collisions Analyze the sort of mishap. In this investigation, we introduced an inventive methodology for fender bender forecast. Methodologically, we showed a learning procedure installed inside a multivariate model can be utilized to distinguish connection between the inspected factors and the car accidents. Future utilizations of this methodology can possibly give bits of knowledge into essential inquiries with respect to street spatial and fleeting unique capacity and viable inquiries in regards to countermeasures.

Future work could incorporate that context oriented data can be utilized to work with object identification in rush hour gridlock scenes and convolutional neural organization can be utilized to create more discriminative element portrayals. We proposed a strategy for shape-based item discovery utilizing distance changes which adopts consolidated courses to fine strategy fit as a fiddle and boundary space too. It works progressively climate with different location objects in a solitary structure strategy.

\section{REFERENCES}

[1]. Abdel-Aty, M., and Abdelwahab, H., Analysis and Prediction of Traffic Fatalities Resulting From Angle Collisions Including the Effect of Vehicles' Configuration and Compatibility. Accident Analysis and Prevention, 2003.

[2]. Abdelwahab, H. T. and Abdel-Aty, M. A., Development of Artificial Neural Network Models to Predict Driver Injury Severity in Traffic Accidents at Signalized Intersections. 
Transportation Research Record 1746, Paper No. 01-2234.

[3]. Bedard, M., Guyatt, G. H., Stones, M. J., \& Hireds, J. P., The Independent Contribution of Driver, Crash, and Vehicle Characteristics to Driver Fatalities. Accident analysis and Prevention, Vol. 34, pp. 717-727, 2002.

[4]. Buzeman, D. G., Viano, D. C., \& Lovsund, P., Car Occupant Safety in Frontal Crashes: A Parameter Study of Vehicle Mass, Impact Speed, and Inherent Vehicle Protection. Accident Analysis and Prevention, Vol. 30, No. 6, pp. 713-722, 1998.

[5]. Dia, H., \& Rose, G., Development and Evaluation of Neural Network Freeway Incident Detection Models Using Field Data. Transportation Research C, Vol. 5, No. 5, 1997, pp. 313-331.

[6]. Evanco, W. M., The Potential Impact of Rural Mayday Systems on Vehicular Crash Fatalities. Accident Analysis and Prevention, Vol. 31, 1999, pp. 455-462.

[7]. Hand, D., Mannila, H., \& Smyth, P., Principles of Data Mining. The MIT Press, 2001.

[8]. Kim, K., Nitz, L., Richardson, J., \& Li, L., Personal and Behavioral Predictors of Automobile Crash and Injury Severity. Accident Analysis and Prevention, Vol. 27, No. 4, 1995, pp. 469-481.

[9]. Kweon, Y. J., \& Kockelman, D. M., Overall Injury Risk to Different Drivers: Combining Exposure, Frequency, and Severity Models. Accident Analysis and Prevention, Vol. 35, 2003, pp. 441-450.

[10]. Martin, P. G., Crandall, J. R., \& Pilkey, W. D., Injury Trends of Passenger Car Drivers In the USA. Accident Analysis and Prevention, Vol. 32, 2000, pp. 541-557.

[11]. Mayhew, D. R., Ferguson, S. A., Desmond, K. J., \& Simpson, G. M., Trends In Fatal Crashes Involving Female Drivers, 1975-1998. Accident
Analysis and Prevention, Vol. 35, 2003, pp. 407415.

[12]. Mussone, L., Ferrari, A., \& Oneta, M., An analysis of urban collisions using an artificial intelligence model. Accident Analysis and Prevention, Vol. 31, 1999, pp. 705-718.

[13]. Ossiander, E. M., \& Cummings, P., Freeway speed limits and Traffic Fatalities in Washington State. Accident Analysis and Prevention, Vol. 34, 2002, pp. 13-18.

[14]. Shankar, V., Mannering, F., \& Barfield, W., Statistical Analysis of Accident Severity on Rural Freeways. Accident Analysis and Prevention, Vol. 28, No. 3, 1996, pp.391-401.

[15]. Sohn, S. Y., \& Lee, S. H., Data Fusion, Ensemble and Clustering to Improve the Classification Accuracy for the Severity of Road Traffic Accidents in Korea. Safety Science, Vol. 4, issue1, February 2003, pp. 1-14.

[16]. Tavris, D. R., Kuhn, E. M, \& Layde, P. M., Age and Gender Patterns In Motor Vehicle Crash injuries: Improtance of Type of Crash and Occupant Role. Accident Analysis and Prevention, Vol. 33, 2001, pp. 167-172.

[17]. Yang, W.T., Chen, H. C., \& Brown, D. B., Detecting Safer Driving Patterns By A Neural Network Approach. ANNIE '99 for the Proceedings of Smart Engineering System Design Neural Network, Evolutionary Programming, Complex Systems and Data Mining, Vol. 9, pp 839-844, Nov. 1999.

[18]. Zembowicz, R. and Zytkow, J. M., 1996. From Contingency Tables to Various Forms of Knowledge in Database. Advances in knowledge Discovery and Data Mining, editors, Fayyad, U. M., Piatetsky-Shapiro, G., Smyth, P., Uthurusamy, R. AAAI Press/The MIT Press, pp.329-349.

[19]. Abraham, A., Meta-Learning Evolutionary Artificial Neural Networks, Neurocomputing Journal, Elsevier Science, Netherlands, Vol. 56c, pp. 1-38, 2004. 
[20]. Moller, A.F., A Scaled Conjugate Gradient Algorithm for Fast Supervised Learning, Neural Networks, Volume (6), pp. 525-533, 1993.

[21]. National Center for Statistics and Analysis http://www-

nrd.nhtsa.dot.gov/departments/nrd-

30/ncsa/NASS.html

[22]. Roh J.W., Bessler D.A. and Gilbert R.F., Traffic fatalities, Peltzman's model, and directed graphs, Accident Analysis \& Prevention, Volume 31, Issues 1-2, pp. 55-61, 1998.

[23]. Peltzman, S., The effects of automobile safety regulation. Journal of Political Economy 83, pp. 677-725, 1975.

[24]. Ossenbruggen, P.J., Pendharkar, J. and Ivan, J., Roadway safety in rural and small urbanized areas. Accid. Anal. Prev. 33 4, pp. 485-498, 2001.

[25]. Abdalla, I.M., Robert, R., Derek, B. and McGuicagan, D.R.D., An investigation into the relationships between area social characteristics and road accident casualties. Accid. Anal. Prev. 29 5, pp. 583-593, 1997.

[26]. Miaou, S.P. and Harry, L., Modeling vehicle accidents and highway geometric design relationships. Accid. Anal. Prev. 25 6, pp. 689709, 1993.

[27]. SVMlight.

http://www.cs.cornell.edu/People/tj/svm_light/. Access date: May, 2003.

[28]. Vapnik, V. N., The Nature of Statistical Learning Theory. Springer, 1995.

[29]. Chong M., Abraham A., Paprzycki M., Traffic Accident Data Mining Using Machine Learning Paradigms, Fourth International Conference on Intelligent Systems Design and Applications (ISDA'04), Hungary, ISBN 9637154302, pp. 415420, 2004.

[30]. Chong M., Abraham A., Paprzycki M., Traffic Accident Analysis Using Decision Trees and Neural Networks, IADIS International Conference on Applied Computing, Portugal,
IADIS Press, Nuno Guimarães and Pedro Isaías (Eds.), ISBN: 9729894736, Volume 2, pp. 39-42, 2004.

[31]. Eui-Hong (Sam) Han, Shashi Shekhar, Vipin Kumar, M. Ganesh, Jaideep Srivastava, Search Framework for Mining Classification Decision Trees, 1996. umn.edu/dept/users/kumar/dmclass.ps

[32]. N. Cristianini and J. Shawe-Taylor, An Introduction to Support Vector Machines, Cambridge University Press, 2000.

[33]. Abraham, Intelligent Systems: Architectures and Perspectives, Recent Advances in Intelligent Paradigms and Applications, Abraham A., Jain L. and Kacprzyk J. (Eds.), Studies in Fuzziness and Soft Computing, Springer Verlag Germany, Chapter 1, pp. 1-35, 2002.

\section{Cite this article as :}

Divya Mishra, Dr Shashank Singh , Dr. Faiyaz Ahamad, "Impact Analysis of Accident Using AI", International Journal of Scientific Research in Computer Science, Engineering and Information Technology (IJSRCSEIT), ISSN : 2456-3307, Volume 7, Issue 4, pp.397-405, July-August-2021. Available at doi $\quad:$ https://doi.org/10.32628/CSEIT217410112 Journal URL : https://ijsrcseit.com/CSEIT217410112 